\title{
Prospective, Longitudinal, Multicenter Observational Study of Long-term Treatment with Intramuscular Interferon $\beta-1$ a of Patients with Clinically Isolated Syndrome at High Risk of Conversion
}

\author{
${ }^{1}$ Hospital Universitario Ntra Sra de la Candelaria, Santa Cruz de Tenerife, Spain \\ ${ }^{2}$ Hospital Clínico Universitario Santiago de Compostela, Santiago de Compostela, Spain \\ ${ }^{3}$ Hospital Virgen de las Nieves, Granada, Spain \\ ${ }^{4}$ Hospital Universitario Reina Sofía, Córdoba, Spain \\ ${ }^{5}$ Hospital Universitario Virgen Macarena, Sevilla, Spain
}

Hernández, Miguel Ángel ${ }^{1 *}$, Prieto, José María², Arnal Carmen ${ }^{3}$, Sánchez Fernando ${ }^{4}$ and Izquierdo Guillermo ${ }^{5}$

\begin{abstract}
Introduction: Early initiation of treatment with intramuscular (IM) interferon (IFN) $\beta$-1a in patients with a single demyelinating event suggestive of multiple sclerosis (MS) has been shown to reduce the risk of a second event. This study investigated the characteristics of patients with clinically isolated syndrome (CIS), conversion to clinically definite $M S$ and quality of life (QoL) in clinical practice.
\end{abstract}

Methods: Patients eligible for IM IFN $\beta-1$ a for CIS were followed for 4 years ( 1 visit every 6 months, 9 visits in total). Disease progression was assessed using the Expanded Disability Status Scale (EDSS). The rate of conversion, adverse events and laboratory parameters were reported. Patients were administered QoL questionnaires (Functional Assessment of Multiple Sclerosis [FAMS] and EuroQol 5D [EQ-5D]).

Results: Thirty-four patients from 15 Spanish hospitals were included and 18 completed the study. The mean \pm SD age of the patients was $32.1 \pm 9.08$ years and $64 \%$ were women. At baseline, the mean EDSS was $0.94 \pm 0.85$. During follow-up, mean EDSS ranged from $0.89-1.25$ and 15 patients (45.5\%) converted to CIS. The most common adverse reaction was flu-like syndrome (55\%). One patient discontinued treatment due to flu-like syndrome.

The mean score on the visual analogue scale of the FAMS and the EQ-5D remained constant throughout the study ( $p>0.05$ with respect to baseline)

Conclusions: The conversion rate was higher than in the immediate-treatment arm of CHAMPIONS study, which could be expected due to differences in the patient population and delays in treatment initiation.

The added value of the present study lies in the prospective collection of data from the clinical practice in highrisk CIS patients treated with IM IFN $\beta-1 a$ in Spain. To our knowledge, this is the first study published with these characteristics.

Keywords: Multiple sclerosis; Clinically isolated syndrome; Intramuscular interferon $\beta$-1a; Conversion rate; Relapses; Treatment compliance; Quality of life

\section{Introduction}

In recent years, our understanding of the early phases of multiple sclerosis (MS) has increased. New imaging techniques make it possible to identify with greater certainty those patients at risk of developing clinically definite MS after an initial demyelinating event suggestive of the disease [1,2].

The better understanding of early phases of the disease prompted studies to investigate whether early interventions could delay the onset of clinically definite MS. For example, in the CHAMPS pivotal study, the effect of intramuscular (IM) interferon (IFN) $\beta$-1a on conversion to clinically definite MS in individuals with a first demyelinating event was compared to placebo [3]. The results were encouraging, with a significantly lower cumulative probability of developing clinically definite MS in the IFN $\beta$-1a group compared to the placebo group over the 3-year follow-up period (rate ratio, 0.49; 95 percent confidence interval, 0.33 to $0.73 ; \mathrm{p}<0.001$ ).

In the CHAMPIONS-5 year study, patients from the CHAMPS study were followed to 5years after the initiation of CHAMPS study [4]. The only factors identified as being independently associated with a lower rate of development of clinically definite MS were randomization to the active treatment (early treatment) and younger age of onset of neurologic symptoms, but the size of the effect was modest. Additional studies of the long-term use of IM IFN $\beta$-1a in patients with clinically isolated syndrome (CIS) in clinical practice, with focus not just on efficacy but also on safety and quality of life (QoL), would provide further support for this indication.

The objective of the present prospective study was to report the rates of conversion to clinically definitive MS and the clinical outcomes of patients with clinically isolated syndrome at high risk of conversion who were treated with IM IFN $\beta$-1a for 4 years. The study also aimed to

*Corresponding author: Hernandez MA, Hospital Universitario Ntra Sra de la Candelaria, Ctra del Rosario, 145. Unidad de Esclerosis Múltiple, $2^{\mathrm{a}}$ Torre Norte. Servicio de Neurología 38010, Spain, Tel: +34 92260 0664; E-mail: mhernandezp78@hotmail.com

Received January 10, 2014; Accepted February 19, 2014; Published February 25,2014

Citation: Hernández, Ángel M, Prieto, María J, Carmen A, Fernando S, et al. (2014) Prospective, Longitudinal, Multicenter Observational Study of Long-term Treatment with Intramuscular Interferon $\beta-1$ a of Patients with Clinically Isolated Syndrome at High Risk of Conversion. J Neurol Neurophysiol 5: 193. doi:10.4172/2155-9562.1000193

Copyright: @ 2014 Hernández, et al. This is an open-access article distributed under the terms of the Creative Commons Attribution License, which permits unrestricted use, distribution, and reproduction in any medium, provided the original author and source are credited. 
Citation: Hernández, Ángel M, Prieto, María J, Carmen A, Fernando S, et al. (2014) Prospective, Longitudinal, Multicenter Observational Study of Long-term Treatment with Intramuscular Interferon $\beta-1$ a of Patients with Clinically Isolated Syndrome at High Risk of Conversion. J Neurol Neurophysiol 5: 193. doi:10.4172/2155-9562.1000193

collect data on the safety and tolerability of IM IFN $\beta$-1a and the QoL in these patients.

\section{Methods}

In this prospective study, conducted in 15 Spanish hospitals between December 2004 and December 2010, patients aged 18-45 years who were eligible for IM IFN $\beta$-1a for CIS were enrolled. Eligibility for initiating treatment after the first demyelinating event was determined according to both the Summary of Product Characteristics (SmPC) and the Spanish Committee for the treatment of MS and CIS patients. However, there were regional differences in the approach to treatment due to differences in the criteria of regional committees. Regional differences in approval criteria are outline below:

Autonomous Communities of Madrid, Murcia, Castilla y León and Asturias: At least 9 T2 lesions and 1 gadolinium enhancing lesion on baseline magnetic resonance imaging (MRI) and 1 new T2 lesion or 1 gadolinium enhancing lesion at 3 months follow-up.

- Autonomous Community of Andalucía: meet one of the two following criteria: 1st. at least $8 \mathrm{~T} 2$ lesions on baseline MRI and positive oligoclonal bands in cerebrospinal fluid and / or immunoglobulin (Ig) G intrathecal secretion. 2nd. at least 8 T2 lesions on baseline MRI and a new T2 lesion or a new gadolinium enhancing lesion at 3 months follow-up.

- Autonomous Community of Galicia: At least 9 T2 lesions on baseline MRI and 1 new T2 lesion or 1 gadolinium enhancing lesion at 3 months follow-up.

- Autonomous Community of Canarias: Avonex ${ }^{\circledR}$ SmPC criteria for the early treatment of MS.

Key exclusion criteria were any previous neurological episode lasting more than 24 hours prior to the one indicated in the inclusion criteria as "first demyelination event" that could be attributed to demyelinization in the judgment of the investigator, pregnancy or breastfeeding in women, immunosuppressive therapy within 6 months prior to the neurological event, a history of hypersensitivity to IFN $\beta$, human albumin or any component of the product Avonex ${ }^{\oplus}$, major depressive disorders or other psychiatric conditions, liver disease, myelo suppression, immune suppression, epilepsy, severe renal or heart disease of any kind, and contraindication of contrast MRI.

Patients remained in the study for 4 years of follow-up only if they did not abandon the treatment within 2 years, switched to another immune modulatory treatment, or developed progressive MS. Other reasons for withdrawal from the study were pregnancy, withdrawal of patient's informed consent and investigator criteria.

We calculated that more than 1000 patients would be eligible for early treatment, based on the incidence of MS in Spain (4-6/100,000), the size of the Spanish population, the ratio patients with a first episode and those with clinically definite MS, and the estimate that $25 \%$ of these patients were high risk.

\section{Follow-up procedures}

Follow-up procedures consisted of 9 visits over the course of 4 years. These visits corresponded to programmed, standard follow-up visits for patients on IM IFN $\beta$-1a in clinical practice. In these visits, patients underwent a neurological examination. Disease progression (on the Expanded Disability Status Scale [EDSS]) and rate of conversion to clinically definite MS were assessed (conversion was defined according to the Poser criteria [5]). Adverse events and laboratory parameters were also reported. To investigate QoL, patients were also administered a disease-specific questionnaire, the Functional Assessment of Multiple Sclerosis (FAMS), whose Spanish version has been validated [6], and a general QoL questionnaire, the EuroQol, also validated in Spanish [7]. With the exception of the QoL questionnaires, the procedures performed at study visits corresponded to those that would be performed in normal clinical practice. Compliance and adherence were assessed by questions about whether injections were performed at the frequency stated in the last visit. If the answer was negative, the researcher inquired about the reasons for noncompliance.

\section{Statistical analysis}

All analyses were performed on an intention-to-treat basis. Study variables were reported using descriptive statistics. Since this was an exploratory study, no formal statistical hypothesis testing was performed. For comparisons with baseline, a Wilcoxon test was used. The SAS statistical package, version 8.2, was used for all analyses.

\section{Results}

\section{Patient population and study disposition}

In total, 34 patients were recruited from 15 hospitals throughout Spain, although 1 patient was excluded because IM IFN $\beta$-1a was not actually administered. Therefore, the study population finally analyzed comprised 33 patients. The demographic and baseline characteristics of these patients are presented in Table 1 . The mean age of subjects on entering the study was 32 years and almost two-thirds were women.

Clinically isolated syndrome had been diagnosed a mean of 1 year before starting treatment. As per the labelling, all patients had received some form of corticosteroid therapy for the initial demyelinating event.

\begin{tabular}{|l|l|}
\hline Demographic characteristics & $32 \pm 9.1$ \\
\hline Age (years) & $12(36.4 \%)$ \\
\hline Sex & $21(63.6 \%)$ \\
\hline Female & $2(6.1 \%)$ \\
\hline Family history of multiple sclerosis & \\
\hline CIS characteristics & $1.0 \pm 0.4$ \\
\hline Duration (years) & $31 \pm 9.2$ \\
\hline Age on diagnosis of CIS (years) & $28(84.8 \%)$ \\
\hline Type of symptoms & $3(9.1 \%)$ \\
\hline Unifocal & $2(6.1 \%)$ \\
\hline Multifocal & $10(30.3 \%)$ \\
Not reported & $6(18.2 \%)$ \\
\hline Type of neurological deficit & $10(30.3 \%)$ \\
Sensitive & $2(6.1 \%)$ \\
Visual & $12(36.4 \%)$ \\
Motor & $1(3.0 \%)$ \\
Myelitis & $3(9.1 \%)$ \\
Brainstem & $33(100 \%)$ \\
\hline Cerebellar & $21(63.6 \%)$ \\
\hline Others & $12(36.4 \%)$ \\
\hline Corticosteroid therapy & \\
\hline Intravenous corticosteroid therapy & \\
\hline Oral corticosteroid therapy & \\
\hline CIS: Clically isolated syndrome & \\
\hline
\end{tabular}

CIS: Clinically isolated syndrome

Data presented as mean \pm SD or $n(\%)$

Table 1: Demographic and baseline characteristics of the patients included in the study. 
Citation: Hernández, Ángel M, Prieto, María J, Carmen A, Fernando S, et al. (2014) Prospective, Longitudinal, Multicenter Observational Study of Long-term Treatment with Intramuscular Interferon $\beta-1$ a of Patients with Clinically Isolated Syndrome at High Risk of Conversion. J Neurol Neurophysiol 5: 193. doi:10.4172/2155-9562.1000193

On inclusion, patients had between 1 and 25 T2 hyper intense lesions (median 10.0), which were mostly supratentorial, and between 0 and $8 \mathrm{~T} 1$ gadolinium enhancing lesions (median 1.0). In the 28 patients with analysis of the cerebrospinal fluid, pathological findings with a cell count $\geq 25$ were found only in one patient. The median IgG index was 0.83 (range, $0.23-5.82$ ) in the 16 patients with an available IgG index determination and oligoclonal bands were found in 23 out of the 25 in which they were analyzed. Visual evoked potentials were assessed in 23 patients and found to be pathological in $10(43.5 \%)$.

Eighteen patients $(54.5 \%)$ completed the 4 -year follow-up period of the study. Ten patients (30\%) withdrew from the study because they switched to another immunomodulator (4 patients due to exacerbations, 1 patient due to tolerability issues and 5 patients due to unknown reasons) while 5 patients withdrew due to pregnancy.

\section{Efficacy}

The median score on the EDSS was 1 throughout the study. The mean EDSS score was $0.94 \pm 0.85$ at baseline visit. During the followup visits, the mean EDSS score fluctuated, ranging from $0.89 \pm 0.83$ (visit 8 ) to $1.25 \pm 0.99$ (visit 7). No clear trend was apparent. The only significant difference with respect to baseline was for visit 3 ( $\mathrm{p}=0.0278$ ).

During follow-up, 15 patients $(45.5 \%)$ had a second relapse (converted to clinically definite multiple sclerosis) and of these, 8 had more than one relapse. Relapses were mostly unifocal (68.2\%) and sensory in nature (59.1\%). Median time between first event suggestive of MS and second event was 20.4 months (max 59.0 months).Mean EDSS score at the time of the first relapse was $0.89 \pm 0.78,0.94 \pm$ 0.85 at baseline visit and $1.11 \pm 0.98$ for those who finished the 4 year follow-up. After conversion, nine of the 15 patients switched to another immunomodulator.

\section{Therapeutic compliance}

Patients were always compliant until visit 5 (24 months) and for the patients who continued on therapy, the percentage of patients always compliant was always above $90 \%$ (Table 2). Considering those patients who withdrew from the study as non-compliant, the percentage of compliant patients was $70 \%$.

\section{Quality of life}

As shown in Figure 1, the different components of the FAMS questionnaire showed little change over time (higher scores indicate worse QoL). Likewise, the overall score showed little variation, with mean values ranging from $60.9 \pm 15.9$ at baseline to $69.8 \pm 27.2$ at visit 9. Of note is that throughout the study, higher scores were obtained for general mood and family and social environment than for the

\begin{tabular}{|c|c|c|}
\hline & Always compliant & Sometimes incompliant \\
\hline Visit 1 $(n=33)$ & $33(100 \%)$ & - \\
\hline Visit 2 $(n=32)$ & $32(100 \%)$ & - \\
\hline Visit 3 $(n=28)$ & $28(100 \%)$ & - \\
\hline Visit 4 $(n=26)$ & $26(100 \%)$ & $2(8.7 \%)$ \\
\hline Visit 5 $(n=23)$ & $21(91.3 \%)$ & $1(4.8 \%)$ \\
\hline Visit 6 $(n=21)$ & $20(95.2 \%)$ & - \\
\hline Visit 7 $(n=20)$ & $20(100 \%)$ & $1(5.3 \%)$ \\
\hline Visit $8(n=19)$ & $18(94.7 \%)$ & \\
\hline Visit 9 $(n=18)$ & $17(94.4 \%)$ & \\
\hline
\end{tabular}

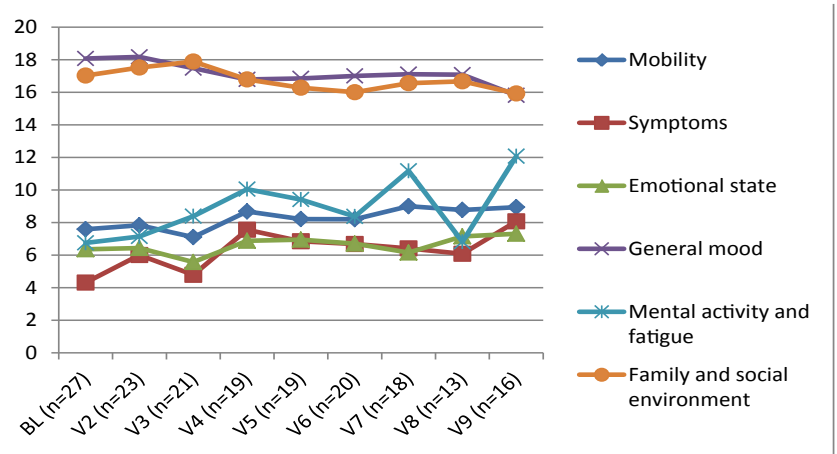

Figure 1: Mean scores on the different components of the Functional Assessment of Multiple Sclerosis (FAMS).

other components. The part of the FAMS questionnaire that deals with "other concerns" (and which does not form part of the overall score) showed that patients were not particularly bothered by the side effects of treatment (mean score never more than 2 at any given visit) while relationship issues and concerns about the disease in general were more worrying (mean score above 3 for components such as "feeling of closeness to your partner", "doctor explains your doubts satisfactorily" and "worried that the disease will worsen"). The non-disease-specific EQ-5D also showed little variation throughout the study (mean score on the visual analogue scale ranged from $84.4 \pm 14.2$ at baseline to 76.7 \pm 17.6 at visit 7 [higher values indicate better QoL]).

\section{Tolerability}

Twenty-nine patients (88.0\%) had at least one adverse drug reaction during the study and, in total, there were 127 adverse drug reactions. These were mainly mild in severity (71\%). Only one severe event was reported (lung cancer, not considered as related to IFN $\beta$ therapy) and only one adverse event led to withdrawal of study drug (flu-like syndrome). Among the adverse events reported, 66.7\% were considered probably, possibly or certainly related to IFN $\beta$ therapy. Most of the reactions considered certainly related were flu-like syndrome and pyrexia.

In the safety laboratory tests, the mean uric acid level did not change significantly between baseline and subsequent visits (ranging from $5.03 \pm 1.41 \mathrm{mg} / \mathrm{dl}$ at the baseline visit to $4.38 \pm 1.38 \mathrm{mg} / \mathrm{dl}$ at visit 5). No more than 3 patients had abnormal values at any given time during follow-up, the same number as at baseline. Few patients had hematological abnormalities (never more than 2 patients per parameter at a given study visit). Alanine transaminase abnormalities were the most frequently reported biochemistry abnormalities throughout the study and indeed such abnormalities were often reported as an adverse drug reaction ( 9 patients [27\%], the second most frequently reported adverse drug reaction after flu-like syndrome). However, these adverse events did not lead to discontinuation of IFN $\beta$ therapy.

\section{Discussion}

Over the last decade, there has been a shift towards earlier treatment in patients considered at risk developing clinically definite MS after a first demyelinating event suggestive of MS (that is, with lesions apparent in brain MRI) [8]. In addition to the CHAMPIONS study with IM IFN $\beta$-1a mentioned earlier [4], studies of early interventions with disease-modifying therapy have been conducted with glatiramer acetate [9], IFN $\beta-1 \mathrm{~b}$ [10] and subcutaneous IFN $\beta-1 \mathrm{a}$ [11]. In all cases, the results supported an early intervention with disease-modifying 
Citation: Hernández, Ángel M, Prieto, María J, Carmen A, Fernando S, et al. (2014) Prospective, Longitudinal, Multicenter Observational Study of Long-term Treatment with Intramuscular Interferon $\beta$-1a of Patients with Clinically Isolated Syndrome at High Risk of Conversion. J Neurol Neurophysiol 5: 193. doi:10.4172/2155-9562.1000193

therapy. However, at the time when this study began (2004), IM IFN $\beta$ la was the only disease-modifying therapy authorized in Spain after a first demyelinating event. The aim of the study was to see when and how frequently a second relapse occurred (clinical conversion to MS) and to assess changes in QoL scales resulting from initiation and maintenance of immunomodulatory therapy in patients at early stage of the disease, before the conversion to clinically definite MS.

In the CHAMPIONS-5 year study, the cumulative probability of development of clinically definite MS was 36\% in immediate treatment group (those randomized to active treatment in the CHAMPS) compared to $49 \%$ in the group who received IFN $\beta$-1a later (those randomized to placebo in CHAMPS and who started active treatment after a median time of 29months) (HR adjusted $=0.57 ; \mathrm{p}=0.008$ ) [4]. This conversion rate is comparatively lower than the $45.5 \%$ conversion rate at 4 years of in our study. Nevertheless, there are substantial differences between our study population and the population in the CHAMPIONS study, in particular the MRI lesion load and the delay in starting treatment. For example, in that study, patients were randomized within 27 days of the first demyelinating event suggestive of MS whereas our patients waited a mean of 1 year before initiating therapy. It is conceivable that an earlier intervention in the case of our study patients would have shown a conversion rate more in line with that found in the "delayed treatment" arm of the CHAMPIONS study. The delay is in part due to the need for all Spanish patients to be evaluated by a local MS committee to ensure eligibility according to the officially approved indication of these committees, which hindered the enrolment since many patients have already converted to clinically definite MS before the committee approved the treatment. While the approval process undoubtedly ensures that individuals are not unnecessarily exposed to treatment, it does delay start of treatment. Ten-year follow-up data are now available for the CHAMPIONS-10 year study [12,13], which further support the need for early intervention (the cumulative probability of developing clinically definite MS was significantly lower in patients who received immediate treatment, with an adjusted hazard ratio of 0.61 [95\% CI, 0.45-0.82], $\mathrm{P}=0.001$ ).

When patients are being treated in the long-term with diseasemodifying therapies, it is important to consider aspects such as adherence to treatment and QoL when assessing the value of treatment, particularly when short-term benefit is often intangible, reducing the motivation to continue to take the therapy. As a result, QoL is receiving increasing recognition as an important outcome in multiple sclerosis therapy $[14,15]$. It is not just physical disability per se, but also other aspects such as cognitive decline and visual impairment that have an impact on QoL [16]. In the early stages of the disease, the effects can be subtle. Concern about the treatment, side effects, compliance, and the impact in the future should also be taken into consideration in order to avoid false expectations. In our study, scores on FAMS, the diseasespecific instrument used in this study, and the generic EuroQoL-5D tended to remain stable. However, worse scores on the FAMS were obtained for general mood and family and social environment than for components pertaining to symptoms or physical aspects of the disease. Patients also expressed concerns about worsening disease.

A stable score on the FAMS questionnaire was also found in the 3 -year follow-up of the BENEFIT study of early versus late IFN $\beta-1 b$ in clinically isolated syndrome [17]. In another study of IFN $\beta$-1a IM in patients with MS, overall QoL did not change significantly over the 12-month follow-up period [18]. Where changes were apparent, these generally correlated with disability progression. As the mean EDSS hardly changed at all in our study, it is not surprising that no changes in QoL were observed.

As mentioned above, adherence to treatment is another important consideration for patients with CIS likely to receive long term immunomodulatory treatment, especially as short-term benefits are often less visible, reducing the motivation to follow the treatment administered. Compliance was generally high in our study, with more than $90 \%$ reporting that they had always been compliant in the preceding 6 months throughout the study. In our study, of 4 years duration, $54.5 \%$ completed the study.

Pregnancy was one of the most common reasons for withdrawal from treatment in our study. MS is a disease that primarily affects young women, many of whom will have a desire to become pregnant [19]. If a woman should become pregnant while taking the drug, the labelling suggests that she be warned of the potential hazards and that discontinuation of the therapy should be considered [20]. Indeed, in our study, becoming pregnant was a withdrawal criteria.

Our study provided a detailed characterization of patients who initiate IFN $\beta$-1a therapy for clinically isolated syndrome in Spain. As required by the labelling, disease activity was apparent in the imaging studies and, of note, almost $93 \%$ of patients had pathological findings in the analysis of cerebrospinal fluid. The presence of oligoclonal bands is thought to be a predictor for developing clinically definite MS [13]. Although not all untreated patients with clinically isolated syndrome develop MS the high prevalence of abnormalities in the cerebrospinal fluid samples confirms that the patients included in our study were generally at a high risk of conversion.

IM IFN $\beta$-1a was well tolerated and the adverse events reported were in line with those described in the SmPC, both in terms of frequency and intensity.

This study is subject to a number of limitations. First and foremost, there is no control arm for our rates of conversion. Nevertheless, the study did provide the opportunity to collect detailed data on the baseline characteristics of patients who entered treatment with IM IFN $\beta-1 b$ in clinical practice. Another weakness is that relatively few patients were enrolled. One explanation for this is the existence of the aforementioned barriers to starting treatment (discussed above). Another possible reason is the heterogeneity of existing criteria in different parts of the country due to the decentralized health system. Such heterogeneity hinders data integrity in an observational study of this type. Of note is that some Spanish regions did not participate in the study and that some patients who were potential candidates for treatment converted to clinically definite MS during the authorization process to initiate treatment so were not finally included in the study.

Without a control arm and with such a small sample size, we cannot make firm affirmations about efficacy. Nevertheless, this study is of interest in that it provides long-term and prospectively collected data in a cohort of CIS patients at high risk of conversion treated in Spain with IM IFN $\beta-1 \mathrm{a}$.

In summary, IM IFN $\beta$-1a was well tolerated and patients showed high levels of compliance. QoL in both the generic and disease-specific questionnaires remained stable in the follow-up period.

\section{Acknowledgement}

The authors would like to thank Biogen Idec Iberia S.L. for financial support and Dr. Gregory Morley for editorial assistance. 
Citation: Hernández, Ángel M, Prieto, María J, Carmen A, Fernando S, et al. (2014) Prospective, Longitudinal, Multicenter Observational Study of Long-term Treatment with Intramuscular Interferon $\beta$-1a of Patients with Clinically Isolated Syndrome at High Risk of Conversion. J Neurol Neurophysiol 5: 193. doi:10.4172/2155-9562.1000193

\section{*AREMIN Study Group}

Arnal C (H. Univ. Virgen de las Nieves, Granada) (5); Izquierdo G, Ruiz J (Compl. H. Virgen Macarena, Sevilla) (5); Sánchez F, Agüera E (H. Reina Sofía Córdoba) (4); Hernández MA, González M (H. Ntra. Sra. De Candelaria, Tenerife)

(3); Oreja C, Díez E, Miralles A, Martínez P (H. la Paz, Madrid) (3); Amigo C (Compl. H. de Pontevedra) (2); Arroyo R, Bartolomé M, de las Heras V (H. Clínico San Carlos, Madrid) (2); García JA, Blasco R (H. Puerta de Hierro, Madrid) (2); Llaneza MA (Compl. H. Arquitecto Marcide, Ferrol) (2); Alemany MJ (H. Doctor Negrín, Palmas de Gran Canaria) (1); Caminero A (H. Ntra. Sra. De Sonsoles, Ávila) (1); Fernández $\mathrm{F}(\mathrm{H}$. de León, León) (1); Fernández-Bolaños R (Compl. H. Ntra. Sra. De Valme, Sevilla) (1); Piñeiro R (Compl. H. Xeral Calde, Lugo) (1); Villaverde R (H. General Univ. J. Morales Meseguer, Murcia) (1); Arias M, Dapena D, Prieto JM (H. Clínico Universitario Santiago de Compostela, Santiago de Compostela) (0).

\section{References}

1. Filippi M, Horsfield MA, Morrissey SP, MacManus DG, Rudge P, et al. (1994) Quantitative brain MRI lesion load predicts the course of clinically isolated syndromes suggestive of multiple sclerosis. Neurology 44: 635-641.

2. Kappos L, Moeri D, Radue EW, Schoetzau A, Schweikert K, et al. (1999) Predictive value of gadolinium-enhanced magnetic resonance imaging for relapse rate and changes in disability or impairment in multiple sclerosis: a meta-analysis. Gadolinium MRI Meta-analysis Group. Lancet 353: 964-969.

3. Jacobs LD, Beck RW, Simon JH, Kinkel RP, Brownscheidle CM et al. (2000) Intramuscular interferon beta-1a therapy initiated during a first demyelinating event in multiple sclerosis. CHAMPS Study Group. N Engl J Med 343: 898-904.

4. Kinkel RP, Kollman C, O'Connor P, Murray TJ, Simon J, et al. (2006) IM interferon beta-1a delays definite multiple sclerosis 5 years after a first demyelinating event. Neurology 66: 678-684.

5. Poser CM, Paty DW, Scheinberg L, McDonald WI, Davis FA, et al. (1983) New diagnostic criteria for multiple sclerosis: guidelines for research protocols. Ann Neurol 13: 227-231.

6. Chang $\mathrm{CH}$, Cella D, Fernández O, Luque G, de Castro P, et al. (2002) Quality of life in multiple sclerosis patients in Spain. Mult Scler 8: 527-531.

7. Badia X, Roset M, Montserrat S, Herdman M, Segura A (1999) [The Spanish version of EuroQol: a description and its applications. European Quality of Life scale]. Med Clin (Barc) 112: 79-85.

8. Tintoré M (2008) Rationale for early intervention with immunomodulatory treatments. J Neurol 255: 37-43.

9. Comi G, Martinelli V, Rodegher M, Moiola L, Bajenaru O, et al. (2009) Effect of glatiramer acetate on conversion to clinically definite multiple sclerosis in patients with clinically isolated syndrome (PreClSe study): a randomised, double-blind, placebo-controlled trial. Lancet 374: 1503-1511.

10. Kappos L, Polman CH, Freedman MS, Edan G, Hartung HP, et al. (2006) Treatment with interferon beta- $1 \mathrm{~b}$ delays conversion to clinically definite and McDonald MS in patients with clinically isolated syndromes. Neurology 67: 1242-1249.

11. Comi G, De Stefano N, Freedman MS, Barkhof F, Polman CH, et al. (2012) Comparison of two dosing frequencies of subcutaneous interferon beta-1a in patients with a first clinical demyelinating event suggestive of multiple sclerosis (REFLEX): a phase 3 randomised controlled trial. Lancet Neurol 11: 33-41.

12. Kinkel RP, Dontchev M, Kollman C, Skaramagas TT, O'Connor PW, et al. (2012) Association between immediate initiation of intramuscular interferon beta-1a at the time of a clinically isolated syndrome and long-term outcomes: a 10-year follow-up of the Controlled High-Risk Avonex Multiple Sclerosis Prevention Study in Ongoing Neurological Surveillance. Arch Neurol 69: 183-190.

13. Miller D, Barkhof F, Montalban X, Thompson A, Filippi M (2005) Clinically isolated syndromes suggestive of multiple sclerosis, part I: natural history pathogenesis, diagnosis, and prognosis. Lancet Neurol 4: 281-288.

14. Williams GC, Rodin GC, Ryan RM, Grolnick WS, Deci EL (1998) Autonomous regulation and long-term medication adherence in adult outpatients. Health Psychol 17: 269-276.

15. Nortvedt MW, Riise T, Myhr KM, Nyland HI (1999) Quality of life in multiple sclerosis: measuring the disease effects more broadly. Neurology 53: 10981103.

16. Rudick RA, Miller DM (2008) Health-related quality of life in multiple sclerosis current evidence, measurement and effects of disease severity and treatment. CNS Drugs 22: 827-839.

17. Kappos L, Freedman MS, Polman CH, Edan G, Hartung H-P, et al. (2007) Effect of early versus delayed interferon beta- $1 \mathrm{~b}$ treatment on disability after a first clinical event suggestive of multiple sclerosis: a 3-year follow-up analysis of the BENEFIT study. Lancet 370: 389-397.

18. Zivadinov R, Zorzon M, Tommasi MA, Nasuelli D, Bernardi M, et al. (2003) A longitudinal study of quality of life and side effects in patients with multiple sclerosis treated with interferon beta-1a. J Neurol Sci 216: 113-118.

19. Sandberg-Wollheim M, Frank D, Goodwin TM, Giesser B, Lopez-Bresnahan M et al. (2005) Pregnancy outcomes during treatment with interferon beta-1a in patients with multiple sclerosis. Neurology 65: 802-806.

20. Summary of Product Characteristics. Avonex®. European Medicines Agency, 2007. 